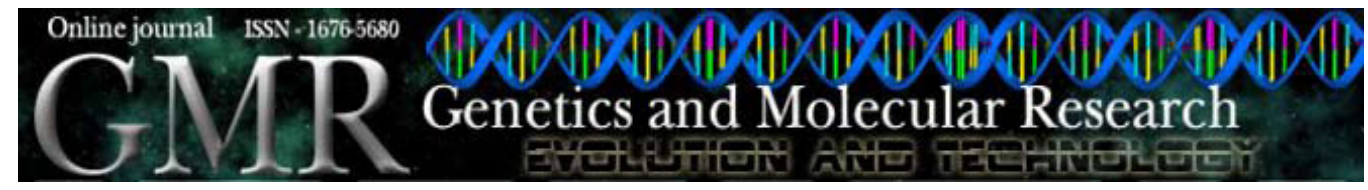

\title{
Association of TIRAP (MAL) gene polymorhisms with susceptibility to tuberculosis in a Chinese population
}

\author{
Y.X. Zhang', Y. Xue ${ }^{1,5}$, J.Y. Liu ${ }^{1,6}$, M.Y. Zhao ${ }^{1}$, F.J. Li ${ }^{2}$, J.M. Zhou ${ }^{3}$, \\ H.J. Wang ${ }^{4}$ and J.C. $\mathbf{L i}^{1}$ \\ ${ }^{1}$ Institute of Cell Biology, Zhejiang University, Zhejiang, China \\ ${ }^{2}$ Hangzhou Red Cross Hospital, Hangzhou, China \\ ${ }^{3}$ Jilin Academy of Traditional Chinese Medicine Sciences, Changchun, China \\ ${ }^{4}$ The Sixth Hospital of Shaoxing, Shaoxing, China \\ ${ }^{5}$ Henan University of Science and Technology, Luoyang, China \\ ${ }^{6}$ Department of Cell Biology, Hangzhou Normal University, Hangzhou, China \\ Corresponding author: J.C. Li \\ E-mail: lijichen@zju.edu.cn
}

Genet. Mol. Res. 10 (1): 7-15 (2011)

Received November 20, 2010

Accepted December 19, 2010

Published January 4, 2011

DOI 10.4238/vol10-1gmr980

\begin{abstract}
Toll-interleukin 1 receptor (TIR) domain containing adaptor protein (TIRAP; also known as MAL) is an essential adaptor molecule in Toll-like receptor signaling, involved in activating the innate immune response during infection. Genetic variations in the TIRAP gene may influence human susceptibility to infectious disease. To date, in the Chinese population, a possible predisposition of TIRAP gene variants to tuberculosis has not been reported. We investigated whether TIRAP gene polymorphisms are associated with the development of tuberculosis in a Chinese population. We investigated all the single-nucleotide polymorphisms (SNPs) within the TIRAP exon 5 in a case-control study of 212 patients with tuberculosis and 215 controls in a Chinese population. Genotyping was performed to identify the polymorphisms of TIRAP gene by PCR-DNA sequencing method. Haplotypes for the TIRAP gene variants were constructed using Haplo-
\end{abstract}


view version 4.2. Six polymorphisms of the SNPs listed in the National Center for Biotechnology Information database were detected in these Chinese tuberculosis patients. It was found that both the frequency of the 286A allele (odds ratio $(\mathrm{OR})=13.37 ; 95 \%$ confidence interval $(\mathrm{CI})=0.75-238.3 ; \mathrm{P}<0.01)$ and the frequency of 286AG genotype $(\mathrm{OR}=13.57 ; 95 \% \mathrm{CI}=0.76-242.5 ; \mathrm{P}<0.01)$ were significantly higher in patients than in healthy controls. However, two other SNPs, C539T and C558T, reported to be associated with tuberculosis in other populations, were found not to be associated with tuberculosis in this Chinese population. We conclude that TIRAP G286A (D96N) polymorphism is associated with susceptibility to tuberculosis and may be a new risk factor for the development of tuberculosis in China.

Key words: Innate immunity; Single-nucleotide polymorphisms; Toll-interleukin 1 receptor domain containing adaptor protein; Disease susceptibility; Tuberculosis

\section{INTRODUCTION}

Tuberculosis (TB) is a contagious and potentially fatal disease caused by various strains of mycobacteria, usually Mycobacterium tuberculosis (Mtb) in humans. It can affect almost any part of the body, but manifests mainly as an infection of the lungs and kills more people each year than any other single infectious disease. Recently, multi-drug resistance of Mtb and extensive-drug-resistance tuberculosis in subjects with compromised immune system have become difficult problems that badly need to be tackled. To effectively control the disease, new drugs, new diagnostics, and more effective vaccines are worldwide priority; they depend on a thorough understanding of the host immune response against Mtb. It is well known that one-third of the world's population is infected with Mtb, but only $10 \%$ among those infected will develop the disease (Rossman and Oner-Eyuboglu, 1998). This fact together with other substantial evidence indicate that variations in host hereditary factors play an important role in susceptibility to TB (Bellamy et al., 2003).

Pattern recognition receptors (PRRs) are the first line of defense against infection in the innate immune system. The core member of PRRs in immune response against Mtb is Toll-like receptors (TLRs) (Akira and Takeda, 2004; Quesniaux et al., 2004). TLRs can recognize pathogen-associated molecular patterns of Mtb and initiate signaling pathways that lead to the activation of the innate immune response, cytokines and formation of the adaptive immune response (Jo, 2008). Mycobacteria are initially recognized by TLR-1, -2, -4, -6, and -9 (Branger et al., 2004; Bafica et al., 2005; Drage et al., 2009; Harding and Boom, 2010; Sanchez et al., 2010), which in turn interact with the adaptor proteins, including MyD88 and Toll-interleukin 1 receptor (TIR) domain containing adaptor protein (TIRAP; also known as MAL) to activate macrophages and dendritic cells (O'Neill and Bowie, 2007). Candidate gene association studies of critical host response genes have successfully revealed that single-nucleotide polymorphisms (SNPs) in TLR-related proteins are significantly associated with infectious and inflammatory diseases (Ogus et al., 2004; Schroder and Schumann, 2005; Hawn et al., 2006; Khor et al., 2007). Interestingly, TIRAP, being the most polymorphic of 
all adapter proteins, contains at least eight SNPs in its coding region. Notable, amongst these polymorphisms is the SNP C539T (rs8177374, also known as S180L), which is a protective factor against tuberculosis and other inflammatory diseases in some ethnic populations (Khor et al., 2007; Castiblanco et al., 2008), but not in others (Nejentsev et al., 2008). Another SNP C558T (rs7932766, also known as A186A), a variant in strong linkage with C539T, was reported to be associated with an increased susceptibility for tuberculosis in a small Vietnamese population (Hawn et al., 2006). Thus, increasing evidence suggests that SNPs in TIRAP gene are important genetic determinants for susceptibility for tuberculosis.

Recently, two similar studies (Nagpal et al., 2009; George et al., 2010) that address the functional and structural consequences of all the SNPs in TIRAP gene-coding region concluded that a rare polymophism in multiple populations, G286A (rs8177400, also known as D96N), acts as a hypomorphic mutation, leading to the loss of function of TIRAP in many aspects. This naturally occurring variant results in impaired cytokine production and NF- $\mathrm{kB}$ activation and interrupts TIRAP to interact with MyD88, after overexpression in HEK 293 and Huh-7 cells (Nagpal et al., 2009; George et al., 2010). Moreover, subsequent population screening of this SNP indicated that it exists in several ethic groups, although with a low frequency of heterozygosity. These data added G286A to the list of functionally important variants of TIRAP and gave evidence that this polymorphism is of epidemiological interest.

China is a country with an alarming TB incidence, reaching more than 300 cases per 100,000 inhabitants in 2003 (Dye, 2006). However, it is unknown whether polymorphisms in TIRAP influence susceptibility to TB in the Chinese. Considering the critical role that TIRAP plays in mediating signals from TLR that recognize Mtb and the evidence of association with TB in other populations, we hypothesized that SNPs in TIRAP affect the susceptibility to TB in the Chinese population. We analyzed the whole coding region sequence of the TIRAP gene and investigated the SNPs and their association with TB in a case-control study in a southeastern Chinese population. To our knowledge, this is the first study to investigate whether polymorphic sites in TIRAP correlate with the development of TB in a Chinese population.

\section{MATERIAL AND METHODS}

\section{Participants}

Two hundred and twelve unrelated patients diagnosed with TB (confirmed by clinical, radiological and bacteriological investigations) were enrolled in the TB group. All TB sufferers were undergoing standard TB treatment at the TB clinic of the Sixth Hospital of Shaoxing and Hangzhou Red Cross Hospital between October 2005 and July 2008. Patients were excluded if they tested positive for human immunodeficiency virus, or were using immunosuppressive agents. The control group comprised 215 healthy, unrelated blood donors with no history of TB or other immune diseases. All control subjects were from the same ethnic (Han) population and geographical origin, and were living in the same region as the patients with TB (Southeast China). This study was approved by the Ethics Committee of the Faculty of Medicine (Zhejiang University, China), and informed consents were obtained from all subjects before blood sampling. 


\section{Genotyping}

The TIRAP gene SNPs were detected by polymerase chain reaction (PCR), followed by direct sequencing. Genomic DNAs were extracted from the peripheral blood leukocytes with the salting-out method (Rousseau et al., 1994). The PCR primer included forward GGAGC AACAGGTCTCTGAGAATAAG and reverse CAAGGCACAGAGCGGGTGAGTAA. The PCR was performed by denaturing at $94^{\circ} \mathrm{C}$ for $3 \mathrm{~min}$, followed by 28 cycles at $94^{\circ} \mathrm{C}$ for 30 $\mathrm{s}, 65^{\circ} \mathrm{C}$ for $45 \mathrm{~s}$, and $72^{\circ} \mathrm{C}$ for $50 \mathrm{~s}$, and a final extension at $72^{\circ} \mathrm{C}$ for $10 \mathrm{~min}$. The amplified products were purified and then identified by scanning with the ABI 3100 sequencer (Applied Biosystems, Carlsbad, CA, USA).

\section{Statistical analysis}

The chi-square test was used to compare allele and genotype distribution in the TB patients and control subjects by the two-tailed Fisher exact method with the GraphPad Prism version 5.01 software. Odds ratios (OR) and 95\% confidence intervals were calculated by Miettinen's method. A P value of $<0.05$ was considered to be significant. Hardy-Weinberg equilibrium was assessed using the chi-square test for each group. Haplotype frequencies and associations were calculated with Haploview version 4.2, which uses the expectation-maximization algorithm (Barrett et al., 2005), from which the D', LOD and $\mathrm{r}^{2}$ calculations were derived. Pairwise linkage disequilibrium was estimated by calculating pairwise $\mathrm{D}^{\prime}$ and $\mathrm{r}^{2}$ based on $\mathrm{D}^{\prime}>0.75$ and $\mathrm{r}^{2}>0.80$.

\section{RESULTS}

Clinical characteristics of 212 TB patients and 215 healthy control subjects were recorded (Table 1). The average age was $38.3 \pm 15.4$ years (range $=18-70$ years) in TB patients and $34.6 \pm 16.2$ years (range $=18-68$ years) in the control group. Females constituted 0.59 of the TB group, and 0.52 of the control group. Similar ratios of males and females were included in each of the two groups $(\mathrm{P}>0.15)$.

Table 1. Characteristics of healthy controls and tuberculosis (TB) patients.

\begin{tabular}{lcc}
\hline & TB group $(\mathrm{N}=212)$ & Control group $(\mathrm{N}=215)$ \\
\hline Age, years range (mean $\pm \mathrm{SD})$ & $18-70(38.3 \pm 15.4)$ & $18-68(34.6 \pm 16.2)$ \\
Gender (female:male) & $87: 125$ & $103: 112$ \\
Pulmonary TB & 191 & $\mathrm{ND}$ \\
Extrapulmonary TB & 21 & $\mathrm{ND}$ \\
Sputum culture-proven & 122 & ND \\
Presence of TB history & 30 & 21 \\
BCG vaccination & 129 & 156 \\
\hline
\end{tabular}

$\mathrm{ND}=$ not determined.

The TIRAP-coding region was amplified and sequenced by PCR in all samples; six polymorphisms were found in this region. All TIRAP SNPs were in Hardy-Weinberg equilibrium except for G164A in the control group (Table 2). 


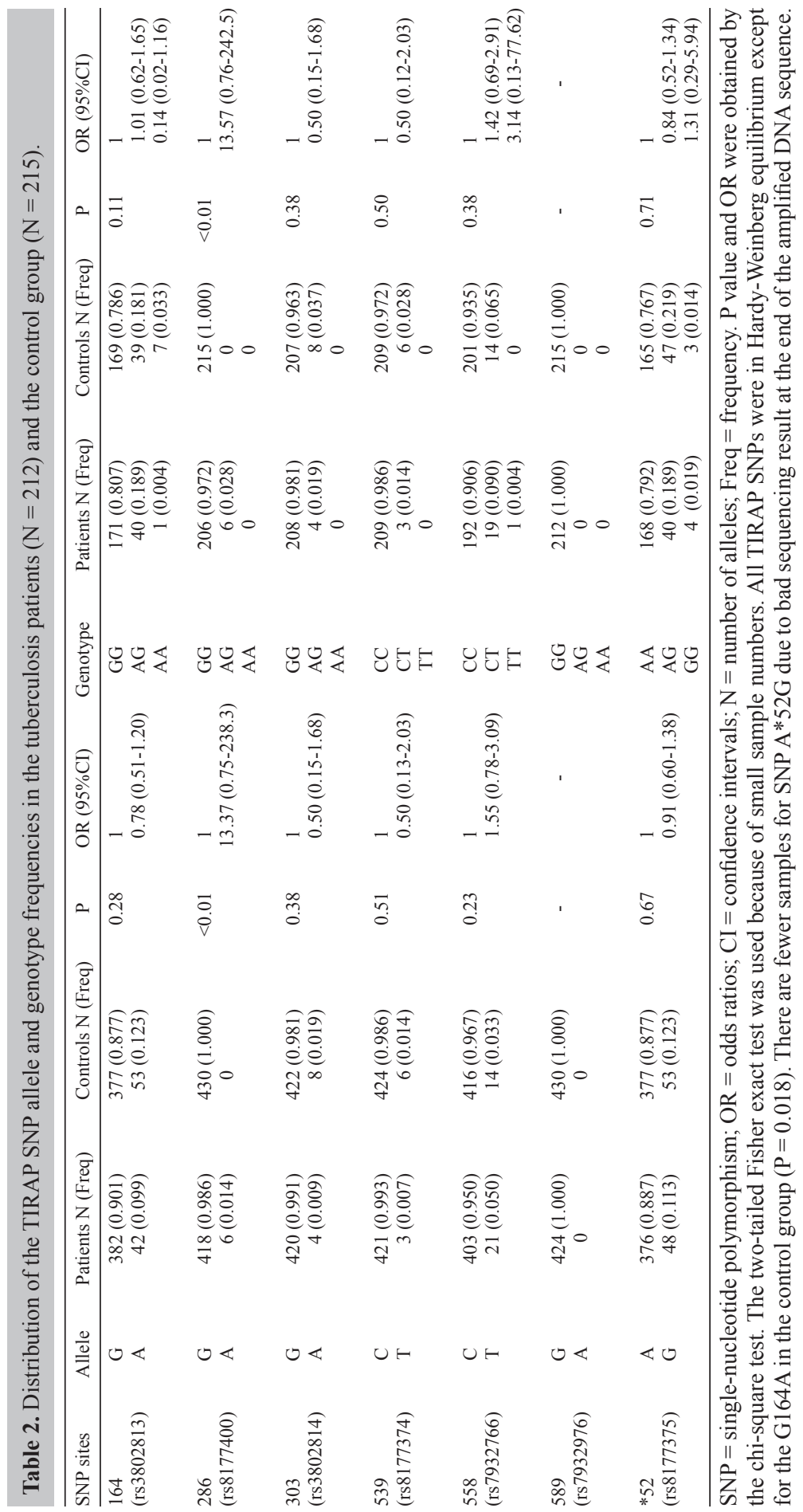


Among all these SNPs, a rare SNP 286A (frequency, 1.4\% in patients and $0 \%$ in controls) was found to be significantly associated with susceptibility to TB (Table 2). Furthermore, the genotype of 286AG also had a significant association with TB (Table 2). SNPs 164A, 303A and 539T had slightly lower frequencies and 558T a higher frequency in the TB group than in controls, but the differences were not significant. No significant differences between patients and controls were observed in the genotype distribution of these SNPs (Table 2).

We further examined haplotypes to analyze whether there were additive associations among the different haplotypes. Only SNPs G303A and C539T were found to be in tight linkage disequilibrium $\left(\mathrm{D}^{\prime}=1\right)$. The GGGCTA haplotype, representing SNPs 164-286-303-539558-*52, was somewhat more frequent in subjects with TB than in controls (Table 3), but no significant difference was found in the frequencies of these haplotypes between the two groups.

\begin{tabular}{|c|c|c|c|c|c|c|c|c|c|c|c|}
\hline \multirow[t]{2}{*}{ Haplotype } & \multicolumn{6}{|c|}{ Allele at marker } & \multicolumn{2}{|c|}{ Haplotype frequency } & \multirow[t]{2}{*}{ Chi-square } & \multirow[t]{2}{*}{$\mathrm{P}$} & \multirow[t]{2}{*}{ OR $(95 \% \mathrm{CI})$} \\
\hline & 164 & 286 & 303 & 539 & 558 & *52 & Patient & Control & & & \\
\hline 1 & $\mathrm{G}$ & $\mathrm{G}$ & G & $\mathrm{C}$ & $\mathrm{C}$ & A & 0.726 & 0.710 & 0.292 & 0.59 & $1.08(0.82-1.43)$ \\
\hline 2 & G & G & G & $\mathrm{C}$ & $\mathrm{C}$ & $\mathrm{G}$ & 0.110 & 0.118 & 0.112 & 0.74 & $0.92(0.56-1.50)$ \\
\hline 3 & A & $\mathrm{G}$ & G & $\mathrm{C}$ & $\mathrm{C}$ & A & 0.094 & 0.118 & 1.278 & 0.26 & $0.78(0.51-1.20)$ \\
\hline 4 & G & G & $\mathrm{G}$ & $\mathrm{C}$ & $\mathrm{T}$ & A & 0.045 & 0.031 & 1.245 & 0.26 & $1.47(0.75-2.89)$ \\
\hline
\end{tabular}

$\mathrm{OR}=$ odds ratios; $\mathrm{CI}=$ confidence intervals.

\section{DISCUSSION}

Some reports present evidence for the role of TIRAP in TB immunity. Yamamoto et al. (2002) reported that lipopolysaccharide (LPS)-induced splenocyte proliferation and cytokine production are abolished and NF-кB and MAPK are induced with delayed kinetics in TIRAP-deficient mice. This suggested a crucial role in the MyD88/TIRAP-dependent signaling pathway shared by TLR-2 and TLR-4, the major pathway for the immune response to Mtb (Jo et al., 2007). Here, we examined the relationship between TIRAP genetic polymorphisms and risk of TB. The main novel finding reported here is that a rare TIRAP polymorphism had a significant influence on the susceptibility for TB and TB-associated TIRAP SNPs in other ethnic groups were found not to impact the susceptibility for TB in this Chinese population.

We found a TIRAP SNP G286A, with a very low allelic frequency of the A allele in TB patients $(1.4 \%)$ and control subjects $(0 \%)$. This is similar to what has been found in other populations: $4.3 \%$ in the European population and $0 \%$ in African Americans (data from NCBI dbSNP). A significant difference was found between patients with TB disease and healthy controls in both TIRAP G286A polymorphism genotype and allelic distribution (Table 2). The risk of developing TB disease in subjects with the 286AG genotype was found to be 13.37fold higher than in carriers of the $286 \mathrm{GG}$ genotype. To our knowledge, this is the first report showing an association between TIRAP SNP G286A and occurrence of TB disease.

It has previously been demonstrated that this TIRAP polymorphism, also known as D96N, resulted in a decrease in the ability of MyD88 binding and TLR-2/TLR-4 signaling. Nagpal et al. (2009) showed that G286A acted as a hypomorphic mutation, with impaired cytokine production and NF-KB activation upon LPS or $\mathrm{PAM}_{2} \mathrm{CSK}_{4}$ stimulation. Moreover, 
co-immunoprecipitation studies and computer modeling data (Nagpal et al., 2009; George et al., 2010) revealed that this variation results in conformation changes in the MyD88 binding site and thus the TIRAP G286A variant is unable to interact with MyD88, a prerequisite for downstream signaling to activate the responses to Mtb. These facts lead us to suggest that the $286 \mathrm{~A}$ allele is a risk factor for increased susceptibility to TB. Nonetheless, only a small part $(1.4 \%)$ of TB patients carried the $286 \mathrm{~A}$ allele. This indicated that other factors, such as other polymorphisms of TIRAP or other TLR-related proteins also contribute to an inability to prevent progression of TB infection to disease. We have reported in a previous study (Xue et al., 2010a) that microsatellite (GT)n polymorphisms in TLR-2 are associated with the susceptibility to TB in Chinese. Data from other studies suggest that polymorphisms of other PRRs are also responsible for human susceptibility to TB (Ogus et al., 2004; Schroder and Schumann, 2005; Barreiro et al., 2006; Ma et al., 2007; Thuong et al., 2007; Austin et al., 2008).

Our results show that other SNPs in the TIRAP gene are not associated with TB in the Chinese population. Several studies have also investigated these SNPs or the linkage disequilibrium of them in TIRAP and their association with TB; however, the results vary significantly among different groups. The TIRAP polymorphism C539T, reported to be a common protective factor against developing TB in West African ( 0.6 and $2 \%$ variation of patients and controls, respectively) (Khor et al., 2007) and Columbian population ( 9 and $16 \%$ variation of patients and controls, respectively) (Castiblanco et al., 2008), was found to be rare (1.4 and $2.8 \%$ variation of patients and controls, respectively), with no significant difference between the patients and controls in our study. Our results are similar to those of some association studies of this variation in other populations, which also found no correlation of SNP with TB in Russian, Ghanaian, Indonesian (Nejentsev et al., 2008), and Vietnamese (Hawn et al., 2006) in a large scale of samples. For the SNP C558T, another study provided evidence showing that both the variant and the haplotype containing the $\mathrm{T}$ allele were more frequent in TB patients than in controls (Hawn et al., 2006). In our study, although both the T allele $(\mathrm{OR}=1.55 ; \mathrm{P}=$ $0.23)$ and the haplotype GGGCTA containing the $\mathrm{T}$ allele $(\mathrm{OR}=1.47 ; \mathrm{P}=0.26)$ were more frequent in patients than controls, no significant difference was found.

There may be several reasons for the differences between these reported results and ours. These discrepant findings could simply represent heterogeneity of association in different populations, which is well described for many immunogenetic polymorphisms (Delgado et al., 2002; Xue et al., 2010b). Worthy of note are the differences in the frequencies of these polymorphisms among different races. The polymorphisms such as G286A and C539T were shown to be much higher in European than in Asian and African populations. This difference, along with gene-gene interactions and environmental and cultural factors, and even the variations in mycobacterium strains, make understanding the observed differences between ethnic groups even more complicated. The relatively small size of the population in our study might be another reason. Given that rare frequencies of the variations and the small size decrease the power to detect small effects, a much larger sample size should provide sufficient variation to determine conclusively the role of TIRAP in TB patients and controls. However, a study (Nejentsev et al., 2008) with a much larger sample, powered enough to detect small effects, also showed a lack of association of the C539T with TB in Russian, Ghanaian and Indonesian populations, which was consistent with our result. Also, the different methods used for identification of alleles and genotypes in different reports may have some influence. Restriction fragment length polymorphisms (RFLP) and allele-specific PCR were used by most of these 
researchers (Khor et al., 2007; Castiblanco et al., 2008; Nejentsev et al., 2008). Here, to avoid the possibility of false or ambiguous results caused by RFLP or allele-specific PCR, we used DNA sequencing to identify gene variation in the TIRAP genes for all the samples.

In conclusion, our data suggested that SNP G286A, which causes an aspartic acid to asparagine substitution at residue 96 of TIRAP, is another candidate gene that may have an influence on susceptibility to TB. Other SNPs of the TIRAP gene, including C539T and C558T, were found to be rare in this southeastern Chinese population and not associated with susceptibility to TB. This is the first report of TIRAP gene polymorphisms screening in Chinese TB patients and provides a new potential genetic risk factor for tuberculosis. Further studies with larger sample sizes are needed to confirm these findings.

\section{ACKNOWLEDGMENTS}

Research supported by grants from Zhejiang Province Special Sci-tech Projects (\#2009C03011-3), National Natural Science Foundation of China (\#81072724), National Special Sci-tech Projects (\#2008ZX10005-010), and the National Fund for Fostering Talents of Basic Science (NFFTBS, \#J0730856, \#J0830833).

\section{REFERENCES}

Akira S and Takeda K (2004). Toll-like receptor signalling. Nat. Rev. Immunol. 4: 499-511.

Austin CM, Ma X and Graviss EA (2008). Common nonsynonymous polymorphisms in the NOD2 gene are associated with resistance or susceptibility to tuberculosis disease in African Americans. J. Infect. Dis. 197: 1713-1716.

Bafica A, Scanga CA, Feng CG, Leifer C, et al. (2005). TLR9 regulates Th1 responses and cooperates with TLR2 in mediating optimal resistance to Mycobacterium tuberculosis. J. Exp. Med. 202: 1715-1724.

Barreiro LB, Neyrolles O, Babb CL, Tailleux L, et al. (2006). Promoter variation in the DC-SIGN-encoding gene CD209 is associated with tuberculosis. PLoS Med. 3: e20.

Barrett JC, Fry B, Maller J and Daly MJ (2005). Haploview: analysis and visualization of LD and haplotype maps. Bioinformatics 21:263-265.

Bellamy R, Fry B, Maller J and Daly MJ (2003). Susceptibility to mycobacterial infections: the importance of host genetics. Genes Immun. 4: 4-11.

Branger J, Leemans JC, Florquin S, Weijer S, et al. (2004). Toll-like receptor 4 plays a protective role in pulmonary tuberculosis in mice. Int. Immunol. 16: 509-516.

Castiblanco J, Varela DC, Castano-Rodriguez N, Rojas-Villarraga A, et al. (2008). TIRAP (MAL) S180L polymorphism is a common protective factor against developing tuberculosis and systemic lupus erythematosus. Infect. Genet. Evol. 8: 541-544.

Delgado JC, Baena A, Thim S and Goldfeld AE (2002). Ethnic-specific genetic associations with pulmonary tuberculosis J. Infect. Dis. 186: 1463-1468.

Drage MG, Pecora ND, Hise AG, Febbraio M, et al. (2009). TLR2 and its co-receptors determine responses of macrophages and dendritic cells to lipoproteins of Mycobacterium tuberculosis. Cell Immunol. 258: 29-37.

Dye C (2006). Global epidemiology of tuberculosis. Lancet 367: 938-940.

George J, Kubarenko AV, Rautanen A, Mills TC, et al. (2010). MyD88 adaptor-like D96N is a naturally occurring lossof-function variant of TIRAP. J. Immunol. 184: 3025-3032.

Harding CV and Boom WH (2010). Regulation of antigen presentation by Mycobacterium tuberculosis: a role for Toll-like receptors. Nat. Rev. Microbiol. 8: 296-307.

Hawn TR, Dunstan SJ, Thwaites GE, Simmons CP, et al. (2006). A polymorphism in Toll-interleukin 1 receptor domain containing adaptor protein is associated with susceptibility to meningeal tuberculosis. J. Infect. Dis. 194: 1127-1134.

Jo EK (2008). Mycobacterial interaction with innate receptors: TLRs, C-type lectins, and NLRs. Curr. Opin. Infect. Dis. 21: 279-286.

Jo EK, Yang CS, Choi CH and Harding CV (2007). Intracellular signalling cascades regulating innate immune responses to Mycobacteria: branching out from Toll-like receptors. Cell. Microbiol. 9: 1087-1098. 
Khor CC, Chapman SJ, Vannberg FO, Dunne A, et al. (2007). A Mal functional variant is associated with protection against invasive pneumococcal disease, bacteremia, malaria and tuberculosis. Nat. Genet. 39: 523-528.

Ma X, Liu Y, Gowen BB, Graviss EA, et al. (2007). Full-exon resequencing reveals Toll-like receptor variants contribute to human susceptibility to tuberculosis disease. PLoS One 2: e1318.

Nagpal K, Plantinga TS, Wong J, Monks BG, et al. (2009). A TIR domain variant of MyD88 adapter-like (Mal)/TIRAP results in loss of MyD88 binding and reduced TLR2/TLR4 signaling. J. Biol. Chem. 284: 25742-25748.

Nejentsev S, Thye T, Szeszko JS, Stevens H, et al. (2008). Analysis of association of the TIRAP (MAL) S180L variant and tuberculosis in three populations. Nat. Genet. 40: 261-262.

O'Neill LA and Bowie AG (2007). The family of five: TIR-domain-containing adaptors in Toll-like receptor signalling. Nat. Rev. Immunol. 7: 353-364.

Ogus AC, Yoldas B, Ozdemir T, Uguz A, et al. (2004). The Arg753GLn polymorphism of the human Toll-like receptor 2 gene in tuberculosis disease. Eur. Respir. J. 23: 219-223.

Quesniaux V, Fremond C, Jacobs M, Parida S, et al. (2004). Toll-like receptor pathways in the immune responses to mycobacteria. Microbes Infect. 6: 946-959.

Rossman M and Oner-Eyuboglu A (1998). Clinical Presentation and Treatment of Tuberculosis. In: Fishman's Pulmonary Diseases and Disorders (Fishman A, ed.). 3rd edn. McGraww Hill Company, New York, 2483-2501.

Rousseau F, Rehel R, Rouillard P, DeGranpre P, et al. (1994). High throughput and economical mutation detection and RFLP analysis using a minimethod for DNA preparation from whole blood and acrylamide gel electrophoresis. Hum. Mutat. 4: 51-54.

Sanchez D, Rojas M, Hernandez I, Radzioch D, et al. (2010). Role of TLR2- and TLR4-mediated signaling in Mycobacterium tuberculosis-induced macrophage death. Cell. Immunol. 260: 128-136.

Schroder NW and Schumann RR (2005). Single nucleotide polymorphisms of Toll-like receptors and susceptibility to infectious disease. Lancet Infect. Dis. 5: 156-164.

Thuong NT, Hawn TR, Thwaites GE, Chau TT, et al. (2007). A polymorphism in human TLR2 is associated with increased susceptibility to tuberculous meningitis. Genes Immun. 8: 422-428.

Xue Y, Jin L, Li AZ, Wang HJ, et al. (2010a). Microsatellite polymorphisms in intron 2 of the Toll-like receptor 2 gene and their association with susceptibility to pulmonary tuberculosis in Han Chinese. Clin. Chem. Lab. Med. 48: 785-789.

Xue Y, Zhao ZQ, Wang HJ, Jin L, et al. (2010b). Toll-like receptors 2 and 4 gene polymorphisms in a southeastern Chinese population with tuberculosis. Int. J. Immunogenet. 37: 135-138.

Yamamoto M, Sato S, Hemmi H, Sanjo H, et al. (2002). Essential role for TIRAP in activation of the signalling cascade shared by TLR2 and TLR4. Nature 420: 324-329. 